\title{
Effect of a Web-Based Guided Self-help Intervention for Prevention of Major Depression in Adults With Subthreshold Depression A Randomized Clinical Trial
}

Claudia Buntrock, MSc; David Daniel Ebert, PhD; Dirk Lehr, PhD; Filip Smit, PhD; Heleen Riper, PhD; Matthias Berking, PhD; Pim Cuijpers, PhD

IMPORTANCE Evidence-based treatments for major depressive disorder (MDD) are not very successful in improving functional and health outcomes. Attention has increasingly been focused on the prevention of MDD.

OBJECTIVE To evaluate the effectiveness of a web-based guided self-help intervention for the prevention of MDD.

DESIGN, SETTING, AND PARTICIPANTS Two-group randomized clinical trial conducted between March 1, 2013, and March 4, 2015. Participants were recruited in Germany from the general population via a large statutory health insurance company (ie, insurance funded by joint employer-employee contributions). Participants included 406 self-selected adults with subthreshold depression (Centre for Epidemiologic Studies Depression Scale score $\geq 16$, no current MDD according to Diagnostic and Statistical Manual of Mental Disorders [Fourth Edition, Text Revision] criteria).

INTERVENTIONS All participants had unrestricted access to usual care (visits to the primary care clinician) and were randomized to either a web-based guided self-help intervention (cognitive-behavioral and problem-solving therapy supported by an online trainer; $\mathrm{n}=202$ ) or a web-based psychoeducation program $(n=204)$.

MAIN OUTCOMES AND MEASURES The primary outcome was time to onset of MDD in the intervention group relative to the control group over a 12-month follow-up period as assessed by blinded diagnostic raters using the telephone-administered Structured Clinical Interview for DSM-IV Axis Disorders at 6- and 12-month follow-up, covering the period to the previous assessment.

RESULTS Among 406 randomized patients (mean age, 45 years; 73.9\% women), 335 (82\%) completed the telephone follow-up at 12 months. Fifty-five participants $(27 \%)$ in the intervention group experienced MDD compared with 84 participants (41\%) in the control group. Cox regression analyses controlling for baseline depressive symptom severity revealed a hazard ratio of $0.59(95 \% \mathrm{Cl}, 0.42-0.82 ; P=.002)$ at 12 -month follow-up. The number needed to treat to avoid 1 new case of MDD was 5.9 ( $95 \% \mathrm{Cl}, 3.9-14.6)$.

CONCLUSIONS AND RELEVANCE Among patients with subthreshold depression, the use of a web-based guided self-help intervention compared with enhanced usual care reduced the incidence of MDD over 12 months. Further research is needed to understand whether the effects are generalizable to both first onset of depression and depression recurrence as well as efficacy without the use of an online trainer.

TRIAL REGISTRATION German Clinical Trial Registry Identifier: DRKS00004709

JAMA. 2016;315(17):1854-1863. doi:10.1001/jama.2016.4326 Last corrected on May 31, 2016.
Supplemental content at jama.com

$+$ CME Quiz at jamanetworkcme.com and CME Questions page 1898

Author Affiliations: Author affiliations are listed at the end of this article.

Corresponding Author: David Daniel Ebert, PhD, Department of Clinical Psychology and Psychotherapy, Friedrich-Alexander University Erlangen-Nuremberg, Nägelsbachstrasse 25a, 90153

Erlangen, Germany

(david.ebert@fau.de). 
$\mathrm{M}$ ajor depressive disorder (MDD) is a highly prevalent condition associated with substantial disease burden and economic costs. ${ }^{1}$ The 12 -month prevalence of MDD in high-income countries is estimated to be $5.1 \%{ }^{2}$ with an annual incidence rate of $3 \% .^{3}$ It is projected that MDD will be the leading cause of premature mortality and disability in high-income countries by $2030 .^{4}$

However, assuming the hypothetical scenario of $100 \% \mathrm{cov}$ erage and adherence to evidence-based treatments, only approximately one-third of the disease burden attributable to MDD could be averted. ${ }^{5}$ Therefore, attention has increasingly been focused on the prevention of MDD. Recent metaanalytic evidence suggests that it is possible to prevent the onset of MDD using psychological interventions by targeting individuals with subthreshold depression (ie, indicated prevention). ${ }^{6}$

Studies, however, were heterogeneous and mostly directed at specific at-risk populations (eg, pregnant women). Targeting at-risk groups becomes less relevant when offering low-cost interventions (eg, web-based interventions). Advantages of web-based interventions include (1) they are accessible at any time and place, (2) participants can work at their own pace and easily review materials, and (3) at-risk individuals are reached at an earlier stage compared with traditional mental health services, because web-based interventions are more easily integrated into daily life. Web-based interventions have been shown effective in reducing depressive symptoms ${ }^{7}$ and acceptable to participants. ${ }^{8}$ To the best of our knowledge, no study has yet investigated the effectiveness of a web-based intervention on the onset of diagnosed MDD. This study evaluated the effect of a web-based guided self-help intervention on the prevention of MDD onset in an adult population with subthreshold depression. An earlier publication from this study reported interim outcomes at posttreatment and 6-month follow-up for depressive symptoms. In this study, we report the primary outcomes from this clinical trial, progression to MDD at 12 months.

\section{Methods}

\section{Trial Design and Participants}

The study protocol is available in Supplement 1. In brief, a 2-group randomized clinical trial was conducted to establish the effectiveness of a web-based guided self-help intervention compared with enhanced usual care on the onset of MDD. The study was approved by the medical ethics committee of the University of Marburg (reference No. AZ 2012-35K) and registered in the German clinical trial registry. Participants provided written informed consent. Study outcomes were assessed at baseline, posttreatment (secondary outcomes only), and 6- and 12-month follow-up.

German citizens are either privately insured (ie, insurers charge a risk-related contribution [11\% of the German population]) or statutorily insured (ie, insurance is funded by joint contributions to sickness funds by employers and employees, based on a percentage of income [89\% of the population]).
Participants were mainly recruited via a large German statutory health insurance company (BARMER GEK) by announcing the study in the company's members' magazine. The BARMER GEK reaches $12.2 \%$ (8.6 million) of the statutorily insured population in Germany. However, adults interested in participating in the study could apply to participate, irrespective of their insurance status. The study was also announced in newspaper articles, on-air media, and related websites. Individuals self-identifying as having a lower mood could apply online on the research website. Referral by a physician was not required.

This open recruitment strategy was chosen to try to approximate the practice setting in which this type of webbased preventive intervention might be used. Applicants were asked to complete an online screening questionnaire to assess whether they (1) experience subthreshold depression (Centre for Epidemiological Studies Depression Scale [CES-D] score $\geq 16),{ }^{9}$ (2) were 18 years or older, (3) had Internet access, (4) were not currently receiving psychotherapy, (5) were not on a waiting list for psychotherapy, (6) had not received psychotherapy in the past 6 months, and (7) did not endorse a notable suicidal risk (Beck Depression Inventory item 9 score $>1$ ). The use of antidepressant medication was not an exclusion criterion because in Germany antidepressants are commonly used for a wide range of indications (ie, depression, anxiety disorders, obsessive-compulsory disorder, chronic pain syndrome, and stress urinary incontinence). ${ }^{10}$ However, participants needed to have been taking a stable dose for at least 4 weeks to be able to enter the study.

Potentially eligible participants were scheduled for a Structured Clinical Interview for Diagnostic and Statistical Manual of Mental Disorders (Fourth Edition) Axis Disorders (SCID) to assess final eligibility, defined as not meeting Diagnostic and Statistical Manual of Mental Disorders (Fourth Edition, Text Revision) (DSM-IV) criteria for (1) a major depressive episode, (2) bipolar disorder, or (3) psychotic disorder, and (4) not having a history of MDD in the past 6 months (based on the model of Kupfer ${ }^{11}$ ). According to Kupfer's model, a patient is considered to be recovered when he or she stays in remission for a minimum of 6 months. In the baseline assessment, participants were asked to self-identify as either white, black, or Hispanic.

\section{Randomization and Masking}

Randomization took place at an individual level and was conducted centrally by an independent researcher not otherwise involved in the study. For the randomization procedure an automated computer-generated random numbers table was used to automatically assign 0 (control group) or 1 (intervention group) to each participant's individual trial ID number. Trial IDs were numbered sequentially and did not entail any specific information about participants (eg, initials). Randomization was performed in the order incoming informed consent forms were received, and the researcher who recruited participants (eg, collected informed consent forms) was not informed about participants' randomization status. Hence, this researcher could not influence the randomization procedure by reordering informed consent 
forms. The researcher conducting the randomization had no information about participants apart from participants' trial ID numbers. Randomization used a block size of 2. Study participants were aware of their allocation. Staff conducting SCID interviews were, however, unaware of participants' randomization status. Steps taken to maintain blinding are described in detail in the study protocol available in Supplement 1.

After each assessment, interviewers were asked to guess each participant's randomization status, and these guesses were compared with the actual status. If blinding was broken during the first outcome interview, the interviewer was replaced with another blinded interviewer for the second outcome interview. The research staff conducting SCID interviews were not otherwise involved in the study.

\section{Interventions}

All study participants had unrestricted access to usual care. For subthreshold depression, usual care entails visits to a primary care clinician but not to treatment provided by a mental health care specialist. The German S3-Guideline/National Disease Management Guideline Unipolar Depression ${ }^{12}$ recommends psychoeducation or more intensive psychological interventions, and the prescription of antidepressant medication, if depressive symptoms intensify (eg, if MDD is diagnosed). In this pragmatic trial, usual care was not protocolized. However, health care utilization was measured with the Trimbos/iMTA Questionnaire For Costs Associated With Psychiatric Illness (TiC-P) ${ }^{13}$ to help develop a description of usual care.

\section{Guided Web-Based Intervention}

The web-based intervention is a multimedia, interactive online tool consisting of six 30-minute sessions. However, the duration of sessions could vary among users. Participants were advised to complete 2 sessions per week if possible and a minimum of 1 . The intervention is based on psychoeducation, behavior therapy, and problem-solving therapy. The content of the intervention is described in detail in the study protocol available in Supplement 1. During the training, participants were supported by an online trainer who provided written individual feedback after each session. Feedback focused on supporting participants to work through the exercises, and no therapeutic advice was provided. Trained graduate students and health care professionals supervised by clinical psychologists provided guidance.

\section{Enhanced Usual Care}

The psychoeducational intervention was based on the German S3-Guideline. ${ }^{12}$ It informed participants about the nature of and evidence-based treatments for depression. The intervention enhanced usual care, in that it systematically offered information that patients might not routinely receive from their primary care clinician. Participants could review the material as often as they desired. However, we did not monitor the actual uptake of the intervention. No online trainer was involved in the intervention, and participants received no homework assignments.

\section{Outcomes}

The primary outcome was time to onset of MDD in the intervention group relative to the control group over a 12-month follow-up period using DSM-IV criteria as assessed with the telephone-administered SCID at 6- and 12-month follow-up, covering the period from the previous assessment. Diagnostic interviews were conducted by psychologists trained in delivering the SCID. The interrater agreement of the axis I disorders is moderate to excellent. ${ }^{14}$ The interformat reliability between face-to-face and telephone-administered SCIDs is considered excellent. ${ }^{15}$ To examine interrater reliability, interviews were audiotaped and second-rated by an independent, blinded, experienced rater. The $\mathrm{\kappa}$ coefficient for interrater agreement for a diagnosis of MDD was 0.77 (based on data from $12 \%$ of the participants), indicating excellent agreement. In case of disagreement between the study interviewer and the independent rater, consensus was reached through discussion.

To reduce potential recall bias, time to onset of MDD was assessed as accurately as possible using the Life Chart method as developed by Lyketsos et al. ${ }^{16}$ In this method, age- and calendar-linked personal landmarks are used to assess the time sequence of, for example, depressive symptomatology and life events in parallel. During the interview, the first day of a depressive episode was established. If the exact day could not be established, the closest week (month) was defined and the midpoint of that week (month) was used.

Secondary clinical outcomes were all based on self-report measures assessed online at 6- and 12-month follow-up and included depressive symptom severity (CES- ${ }^{9}$ ), functional impairment (12-Item Short Form Health Survey $[\mathrm{SF}-12]^{17}$ ), anxiety (Hospital Anxiety and Depression ScaleAnxiety [HADS-A $]^{18}$ ), problem-solving skills (Social ProblemSolving Inventory-Revised [SPSI-R $]^{19}$ ), behavioral activation (Behavioral Activation for Depression Scale-Short Form [BADS-SF ${ }^{20}$ ), mastery (Pearling and Schooler Mastery Scale $[\mathrm{PSMS}]^{21}$ ), worrying (Penn State Worry Questionnaire $[\mathrm{PSWQ}]^{22}$ ), insomnia severity (Insomnia Severity Index $[\mathrm{ISI}]^{23}$ ), and health care service uptake $\left(\mathrm{TiC}-\mathrm{P}^{13}\right)$.

\section{Statistical Analysis}

Because no estimate of clinical relevance exists for the incidence of MDD, we assumed an absolute risk reduction of at least $10 \%$ for the incidence of MDD between the intervention and control groups as clinically relevant. This (normative) threshold was derived by consulting clinical experts in the field of depression prevention and asking stakeholders who would potentially use a web-based intervention in routine care (eg, health insurance companies) about a threshold above which they would consider the results worthwhile from a clinical perspective. Based on previous studies evaluating interventions directed at the prevention of MDD, we expected a $25 \%$ mean incidence of MDD in the control group within the 12-month follow-up period. ${ }^{24,25}$

A power calculation indicated that 406 participants were needed to demonstrate an absolute risk reduction of $10 \%$ between the conditions as statistically significant at $\mathrm{a}<.05$ (2-tailed) 
with a power of $(1-\beta)=0.80$ using survival analysis while accounting for $20 \%$ dropout (calculated using PASS 12 [NCSS]).

All analyses are reported according to the CONSORT statement. ${ }^{26}$ Analyses were based on intention to treat; ie, all randomized participants were included in the analyses, irrespective of whether they adhered to the treatment protocol. Kaplan-Meier curves and Cox proportional hazard regression analyses were used to determine differences in time to onset of MDD (in weeks) between the intervention and control groups. ${ }^{27}$ The mean survival time was calculated as the area under the Kaplan-Meier survivor function within the 12-month trial period. Cox regression computes estimates of survival time for right-censored data. An observation is rightcensored when a participant is no longer eligible to experience a depressive episode, eg, the participant is lost to follow-up or completes the follow-up period without experiencing a major depressive episode. Time to onset of MDD was used as the dependent variable and treatment condition as the independent variable covarying baseline depressive symptom severity (ie, baseline CES-D sum score). Concurrent use of antidepressants was also included as a covariate in the Cox proportional hazards model (post hoc). Because the use of antidepressants was not a predictor of the outcome, it was excluded from the final model. The Cox model assumes that hazards are proportional, implying that the effect of a given covariate does not change over time. We tested the proportional hazards assumption based on the scaled Schoenfeld residuals test. ${ }^{28}$

Person-time-based Poisson regression was used to obtain incidence rate ratios (IRRs). We calculated the number needed to treat (NNT) and 95\% CIs to avoid 1 additional case of MDD as compared with the control group, according to Altman and Andersen. ${ }^{29}$

In secondary analyses, analysis of covariance was used to compare outcomes between groups at 12-month follow-up, adjusting for baseline scores to assess differences in secondary outcomes. The effects on secondary clinical outcomes at posttreatment and 6-month follow-up have been reported. ${ }^{30}$

Missing data were imputed using multiple imputation techniques as implemented in Stata 13 (StataCorp). In addition, perprotocol analyses were conducted based on the sample of participants who adequately adhered to the intervention protocol (ie, completed at least 5 of 6 intervention sessions). A significance level of .05 (2-sided) was used for all outcome analyses. All data were analyzed using Stata 13 for Windows. ${ }^{31}$

\section{Sensitivity Analyses}

To test the robustness of the findings, missing data were imputed using imputation techniques for survival data as implemented in Stata 13, and the same Cox model as that described for the main analyses was used. In addition, we tested the robustness of the findings by excluding those participants who revealed their randomization status during SCID follow-up interviews. To assess the robustness of the preventive effect of the intervention, a subgroup analysis excluded those participants taking antidepressants at baseline. A per-protocol analysis tested whether intervention completers (ie, those completing at least 5 of 6 intervention sessions) differed from noncompleters with regard to any baseline characteristics (eTable 1 in Supplement 2) and with regard to time to onset of a major depressive disorder.

\section{Results}

\section{Participant Characteristics}

Figure 1 shows the flow of participants through the study. Between March 15, 2013, and March 4, 2014, the study enrolled 406 participants (intervention group, $n=202$; control group, $n=204)$. Overall, 335 participants (82\%) participated in the SCID follow-up interviews (69 participants were censored at baseline and 2 at 6-month follow-up). There were no significant differences in follow-up rates between study conditions ( $P=.22$ by $X^{2}$ test). Dropout was not associated with baseline depressive symptom severity or any sociodemographic factor.

Some participants ( $\mathrm{n}=17$ [5.1\%]) informed interviewers about their randomization status during the SCID follow-up interviews (10 in the intervention group, 7 in the control group). Interviewers correctly guessed a participant's randomization status in 53\% of cases. Participant characteristics at baseline are described in detail elsewhere ${ }^{30}$ and shown in Table 1. In brief, the modal participant was female, white, and 45 years old, had an above-average level of education, and was employed.

\section{Effectiveness of Intervention}

The mean treatment duration was 5.84 (SD, 4.37) weeks. On average, participants completed 4.93 of 6 sessions. ${ }^{30}$ The total time a trainer spent per participant was approximately 3 hours. Fifty-five participants (27\%) in the intervention group and 84 participants (41\%) in the control group experienced MDD. Figure 2 shows the Kaplan-Meier survival curves for the intervention and control groups generated for the 12-month study period. The Kaplan-Meier estimates of the cumulative incidence of MDD were 32\% (95\% CI, 25\%$39 \%$ ) for the intervention and $47 \%$ (95\% CI, 40\%-55\%) for the control condition. The corresponding person-time-based IRR was 0.60 (95\% CI, 0.42-0.84; $P=.003)$. The log-rank test revealed a statistically significant difference between incidence rates over time $(P=.002)$. The mean time to onset of MDD within the 12-month trial period was 43 weeks (95\% CI, 41-46) in the intervention group and 37 weeks (95\% CI, 35-40) in the control group. Cox regression, which controlled for baseline depressive symptom severity, revealed a hazard ratio [HR] of 0.59 ( $95 \% \mathrm{CI}, 0.42-0.82 ; P=.002)$. The estimated HR for depressive symptom severity was 1.06 (95\% CI, 1.04-1.08; $P<.001$ ). There was no evidence for nonconstant HRs (global test of nonproportionality, $P=.97$; treatment condition, $P=.90$; depressive symptom severity, $P=.84)$.

At 12-month follow-up, the NNT to avoid 1 new case of MDD was 5.9 (95\% CI, 3.9-14.6). In the sensitivity analysis using imputed data instead of censoring for missing values, Cox regression (again controlling for baseline depressive symptom severity) revealed an $\mathrm{HR}$ of 0.65 (95\% CI, 0.49-0.86; $P=.002$ ) 


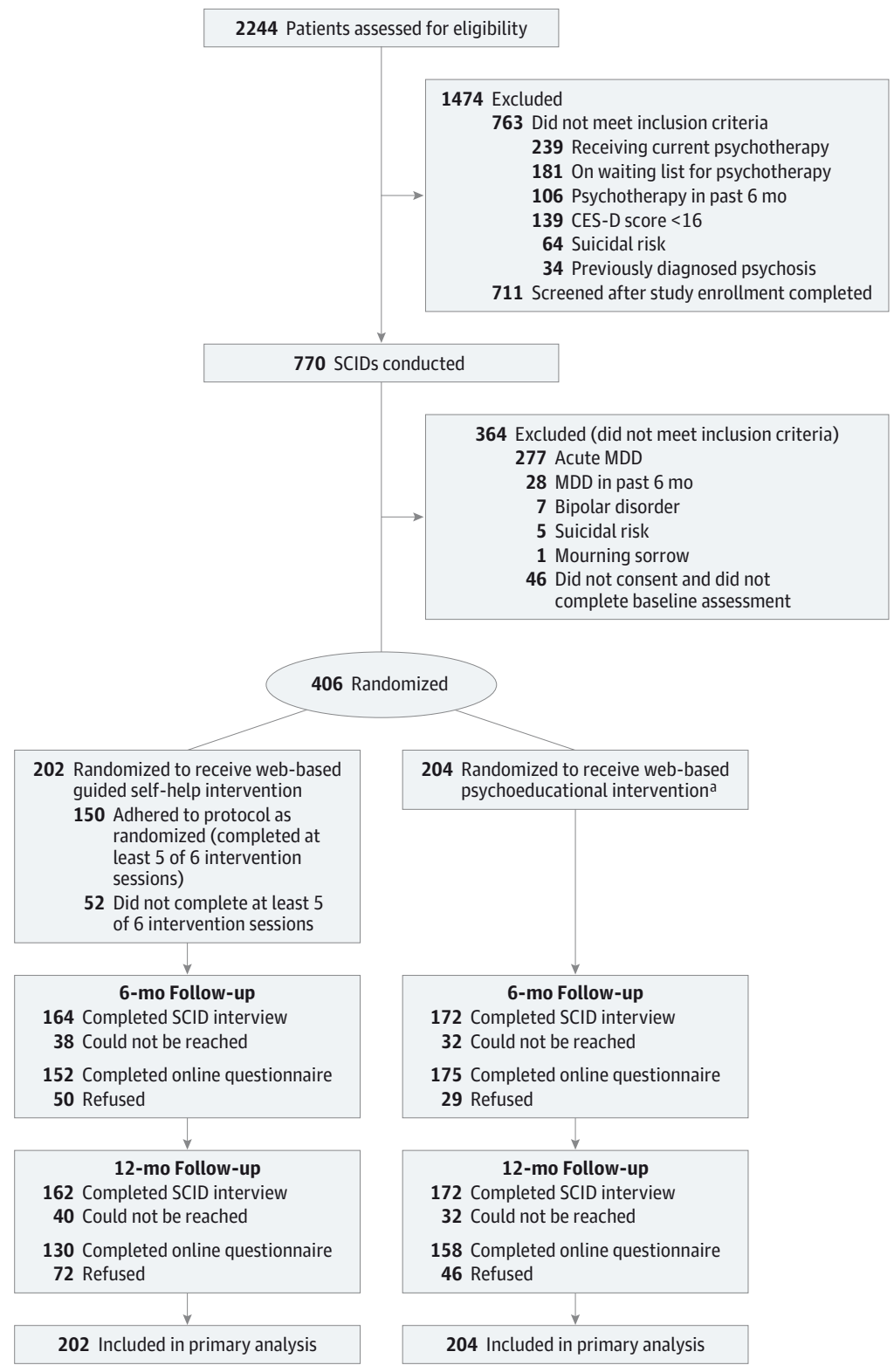

Adapted from Buntrock et al. ${ }^{30}$ CES-D indicates Centre for Epidemiological Studies Depression Scale; MDD, major depressive disorder; SCID, Structured Clinical Interview for Diagnostic and Statistical Manual of Mental Disorders (Fourth Edition) Axis Disorders.

${ }^{a}$ Adherence to the intervention could not be assessed. (see also eFigure in Supplement 2). Excluding participants taking antidepressants at baseline or those who revealed their randomization status during the SCID interview resulted in HRs similar to those from the main analysis (Supplement 2). Detailed results of the sensitivity and per-protocol analyses are reported in Supplement 2.

\section{Secondary Outcomes}

Table 2 reports means, 95\% CIs, and between-group effect sizes of secondary clinical outcomes at follow-up assessments based on the intention-to-treat sample.

Significant differences in change from baseline to 12month follow-up in favor of the intervention group were found for all outcomes except for the physical health summary score of the SF-12, the positive problem-orientation subscale of the
SPSI-R, and worrying (PSWQ). Corresponding effect sizes were small to moderate (Table 2). We did not find any significant differences in health care use (ie, outpatient care, inpatient care, or use of antidepressants) between study conditions (Table 3).

\section{Discussion}

We examined whether a web-based guided self-help intervention was effective in preventing the onset of diagnosed MDD when compared with enhanced usual care over a 12-month follow-up period in patients experiencing subthreshold depression. Results of the study suggest that the intervention could effectively reduce the risk of MDD onset or at least delay onset. 
Table 1. Baseline Characteristics of Participants Experiencing Subthreshold Depression According to Study Group ${ }^{\text {a }}$

\begin{tabular}{|c|c|c|c|}
\hline \multirow[b]{2}{*}{ Characteristic } & \multicolumn{3}{|l|}{ №. (\%) } \\
\hline & $\begin{array}{l}\text { Intervention Group } \\
(\mathrm{n}=202)\end{array}$ & $\begin{array}{l}\text { Control Group } \\
(n=204)\end{array}$ & $\begin{array}{l}\text { Total Sample } \\
(\mathrm{n}=406)\end{array}$ \\
\hline CES-D sum score, mean (SD) & $26.25(7.85)$ & $26.42(7.99)$ & $26.34(7.91)$ \\
\hline Age, mean (SD) & $45.71(11.93)$ & $44.38(11.84)$ & $45.04(11.89)$ \\
\hline \multicolumn{4}{|l|}{ Sex } \\
\hline Men & $53(26.2)$ & $53(26)$ & $106(26.1)$ \\
\hline Women & $149(73.8)$ & $151(74)$ & $300(73.9)$ \\
\hline \multicolumn{4}{|l|}{ Relationship } \\
\hline Single & $62(30.7)$ & $67(32.8)$ & $129(31.8)$ \\
\hline Married or cohabiting & $102(50)$ & 107 (52.9) & $209(51.5)$ \\
\hline Divorced or separated & $37(18.3)$ & $25(12.3)$ & $62(15.3)$ \\
\hline Widowed & $2(1)$ & $4(2)$ & $6(1.5)$ \\
\hline \multicolumn{4}{|l|}{ Ethnicity } \\
\hline White & $165(81.2)$ & $174(85.8)$ & $339(83.5)$ \\
\hline Black & $1(0.5)$ & 0 & $1(0.2)$ \\
\hline Hispanic & 0 & $1(0.5)$ & $1(0.2)$ \\
\hline Not reported & $37(18.3)$ & $28(13.7)$ & $65(16)$ \\
\hline \multicolumn{4}{|l|}{ Level of education } \\
\hline Low, primary ${ }^{b}$ & $5(2.5)$ & $3(1.5)$ & $8(2)$ \\
\hline Middle, secondary ${ }^{c}$ & $33(16.3)$ & $34(16.7)$ & $67(16.5)$ \\
\hline High, A-level or higher ${ }^{d}$ & $164(81.2)$ & 167 (81.9) & $331(81.5)$ \\
\hline \multicolumn{4}{|l|}{ Employment status } \\
\hline Full-time working & $105(52)$ & $106(52)$ & $211(52)$ \\
\hline Part-time working & $65(32.2)$ & 59 (28.9) & $124(30.5)$ \\
\hline Nonworking & $26(12.4)$ & $28(14.2)$ & $54(13.3)$ \\
\hline Unemployed or seeking work & $4(2)$ & $8(3.9)$ & $12(3)$ \\
\hline On sick leave & $3(1.5)$ & $2(1)$ & $5(1.2)$ \\
\hline \multicolumn{4}{|l|}{ Income, $€^{e}$} \\
\hline Low, $<10000$ & $16(7.9)$ & $25(12.3)$ & $41(10.1)$ \\
\hline Middle, $10000-60000$ & $145(71.8)$ & $149(73)$ & $294(72.4)$ \\
\hline High, >60000 & $26(12.9)$ & $12(5.9)$ & $38(9.4)$ \\
\hline Not reported & $18(8.8)$ & $15(7.4)$ & $33(8.1)$ \\
\hline \multicolumn{4}{|l|}{ Previous } \\
\hline Psychotherapy ${ }^{f}$ & $88(43.6)$ & $88(42.2)$ & $176(43.4)$ \\
\hline Health training ${ }^{g}$ & $51(25.2)$ & $45(22.1)$ & $96(23.6)$ \\
\hline Use of antidepressants & $50(24.8)$ & $44(21.6)$ & $94(23.2)$ \\
\hline \multicolumn{4}{|l|}{ Way of recruitment } \\
\hline Health insurance company & $91(45)$ & $94(46.1)$ & $185(45.6)$ \\
\hline Press articles or Internet search & $70(34.7)$ & $73(35.8)$ & $143(35.2)$ \\
\hline Not known & $41(20.3)$ & $37(18.1)$ & $78(19.2)$ \\
\hline
\end{tabular}

Abbreviation: CES-D, Center for Epidemiologic Studies Depression Scale.

${ }^{\text {a }}$ Adapted from Buntrock et al. ${ }^{30}$

b Primary education indicates elementary school.

c Secondary education indicates high school.

dIndicates A-level examinations ("Abitur") or above (university degree)

e Yearly gross income

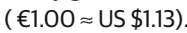

${ }^{\mathrm{f}}$ Ever in life but not in preceding 6 months before entering the study.

${ }^{g}$ Preventive interventions as offered by German statutory health insurance companies (eg, stress management, smoking cessation, healthy diet).
The incidence of MDD in the control group was remarkably higher than that usually found in prevention studies. ${ }^{6}$ The possibly substantial secondary prevention population may have been a reason for the high rate of MDD in the control group. However, some prevention studies assessed only current MDD at follow-up, hence not covering the whole study time frame. ${ }^{24,32}$ The HR of 0.59 (95\% CI, 0.42-0.82) found in the present study compares favorably with results from other indicated prevention studies focusing on an adult population without additional risk factors. To our knowledge, only 2 such studies on non-web-based interventions have been conducted so far and reveal mixed results, with
IRRs ranging from 0.66 (95\% CI, 0.40-1.09) $)^{33}$ to 1.07 (95\% CI, $0.57-2.01) \cdot{ }^{34}$ Results of the present study are also comparable with preventive effects of psychological interventions in at-risk populations (eg, HR, 0.60 [95\% CI, 0.31-1.16] in patients with physical illness). ${ }^{35}$ Indicated preventive interventions could thus also target populations without additional risk indicators to result in clinically relevant effects. Considering the high incidence rate in the control group, the intervention might particularly attract those participants with an increased risk of developing MDD.

The present study may have implications for clinical practice and research. First, to our knowledge, this trial is 
Figure 2. Kaplan-Meier Survival Estimates of Time to Onset of Major Depressive Disorder by Study Group

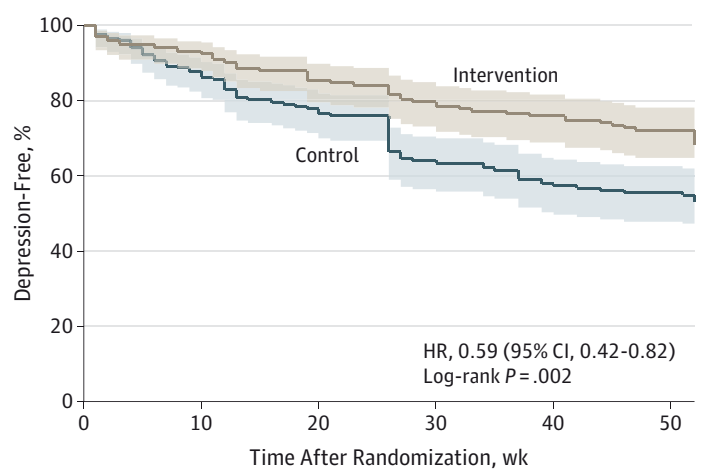

No. at risk

$\begin{array}{llllll}\text { Intervention } 202 & 188 & 136 & 125 & 119 & 113\end{array}$

$\begin{array}{lrrrrrr}\text { Control } & 204 & 179 & 129 & 106 & 96 & 92\end{array}$

The primary end point was time to onset (in weeks) of a major depressive disorder in the intervention relative to the control group according to Structured Clinical Interview for Diagnostic and Statistical Manual of Mental Disorders (Fourth Edition) Axis Disorders criteria. Follow-up time was truncated at 52 weeks. The log-rank test and Cox proportional hazard regression analysis controlling for baseline depressive symptom severity were used to test for group differences. HR indicates hazard ratio. The shaded areas illustrate $95 \% \mathrm{Cls}$.

the largest prevention trial conducted so far, and it supports the effectiveness of indicated preventive interventions. Second, the NNT of 5.9 found in the present study is comparable with NNTs from studies of the treatment of MDD. ${ }^{36}$ It implies that of those patients identified with subthreshold depression and participating in the intervention, almost $17 \%$ would benefit in terms of a prevented episode of MDD within a 12-month period. Third, of participants who developed MDD within the 12-month trial period, the onset of MDD was delayed in the intervention group as compared with the control group. Although preventing the onset of depressive episodes is preferable because it results in complete avoidance of disease burden, delaying the onset of MDD is also important. Every year in which new cases of MDD could be avoided will result in both considerably less pain (to the patients themselves and also their families) and reduced economic costs.

Fourth, this study revealed that reducing the incidence of MDD is also possible using a web-based guided self-help intervention. Fifth, web-based interventions might attract people who may not use face-to-face interventions. Less burdensome interventions are needed, because the majority of individuals experiencing depressive symptoms do not seek help, ${ }^{37}$ and participation rates in face-to-face interventions for subthreshold depression are low. ${ }^{38}$ Delivering low-threshold evidence-based preventive interventions via the Internet may be a strategy with potential to reach individuals at an early stage and may help to prevent the transition from subthreshold depression to a full-blown depressive disorder or relapses in recurrent depressive disorder. However, the applicability of webbased interventions is related to the acceptance of such interventions by the target population (eg, preferences for dif- ferent treatment modalities, such as face-to-face interventions) and the availability of technical resources (eg, reliable access to the Internet).

However, this study has some limitations. First, we did not assess lifetime history of MDD at baseline, meaning that the results of this study refer to a mixed sample of first depression onsets and recurrences. The incidence rate in the control group was higher than could be expected if predominantly participants without lifetime history of depression were included. Thus, we cannot conclude whether results can be generalized to both first onset of depression and prevention of recurrence. Future studies should thus clarify whether web-based guided self-help interventions are effective both for the prevention of first depression onset and the prevention of recurrence. Second, the time horizon of this study was limited to 12 months. Third, randomization with a block size of 2 was used. It would appear that randomization with a block size of 2 ensures that whoever performed the randomization knew in advance the allocation of half of the participants. However, the randomization procedure was performed in the order that incoming informed consent forms were received, and collection of informed consent forms and randomization of participants were performed by 2 independent researchers using procedures designed to preserve the concealment of allocation.

Fourth, we identified a $10 \%$ risk reduction as clinically relevant. However, this assumption was based on expert opinion. Fifth, we did not assess chronic medical conditions. Experiencing chronic medical conditions might be a risk indicator for the onset of MDD. Future studies should thus assess such conditions or evaluate the effects of web-based guided selfhelp interventions directly in such patient groups. Sixth, it was not possible to mask participants to their assigned study condition. This is a common problem in trials evaluating psychological interventions. Nevertheless, it might have distorted results of the trial.

Seventh, not all individuals may benefit from this particular web-based intervention to the same degree. Future studies should investigate potential effect modifiers (eg, Internet literacy). Eighth, usual care was not standardized across primary care clinicians. Because primary care clinicians were not known to us, we could not adjust for clinician in the analyses. Ninth, participants in this study were better educated than the general population and predominantly women. Conclusions drawn from the present study may therefore not generally apply to other populations. However, in this trial we used an open recruitment strategy that mimicked the way in which people likely will be recruited for e-health interventions in the future, thus providing ecological validity to the current study and the sample on which it is based.

Tenth, although the control group received access to a webbased psychoeducational intervention, the study conditions were not balanced with regard to human support. This method was chosen because we wanted to evaluate the effects of the intervention compared with usual care, the comparator usually used in pragmatic trials aiming to achieve high ecological validity. ${ }^{39}$ However, we cannot rule out that part of the observed preventive effect is caused by human attention. 
Table 2. Means $(95 \% \mathrm{Cl})$ and Between-Group Effect Sizes for Each Secondary Clinical Outcome Measure and Measurement Based on the Intention-to-Treat Sample (Imputed Data)

\begin{tabular}{|c|c|c|c|}
\hline \multirow[b]{2}{*}{ Measure } & \multicolumn{2}{|l|}{ Mean $(95 \% \mathrm{Cl})$} & \multirow{2}{*}{$\begin{array}{l}\text { Between-Group Effect Size } \\
\text { Cohen d }(95 \% \mathrm{Cl})\end{array}$} \\
\hline & Baseline Assessment & 12-Month Follow-up & \\
\hline \multicolumn{4}{|l|}{ CES-D } \\
\hline Intervention & 26.26 (25.17 to 27.35$)$ & 16.84 (15.96 to 17.99$)$ & \multirow{2}{*}{$0.29(0.09$ to 0.04$)$} \\
\hline Control & $26.42(25.32$ to 27.53$)$ & 19.42 (18.08 to 20.76$)$ & \\
\hline \multicolumn{4}{|l|}{ SF-12 } \\
\hline \multicolumn{4}{|l|}{ MCS } \\
\hline Intervention & 31.45 (30.39 to 32.43$)$ & 43.50 ( 42.22 to 44.80$)$ & \multirow{2}{*}{0.37 (0.17 to 0.56$)$} \\
\hline Control & 30.77 (29.57 to 31.76$)$ & 39.86 (38.40 to 41.32$)$ & \\
\hline \multicolumn{4}{|l|}{ PCS } \\
\hline Intervention & 47.47 (46.12 to 48.82 & 48.42 ( 47.31 to 49.52$)$ & \multirow{2}{*}{$0.07(-0.12$ to 0.26$)$} \\
\hline Control & 47.83 (46.53 to 49.03$)$ & 47.86 (46.76 to 48.96$)$ & \\
\hline \multicolumn{4}{|l|}{ HADS-A } \\
\hline Intervention & 9.59 (9.14 to 10.05$)$ & 6.63 (6.17 to 7.09$)$ & \multirow{2}{*}{$0.34(0.15$ to 0.54$)$} \\
\hline Control & 9.59 (9.12 to 10.01$)$ & 7.83 (7.33 to 8.33$)$ & \\
\hline \multicolumn{4}{|l|}{ BADS-SF } \\
\hline Intervention & 25.45 (24.37 to 26.53$)$ & 33.93 (32.84 to 35.03$)$ & \multirow{2}{*}{$0.34(0.14$ to 0.53$)$} \\
\hline Control & 24.62 (23.57 to 25.66$)$ & 31.25 (30.14 to 32.37$)$ & \\
\hline \multicolumn{4}{|l|}{ SPSI } \\
\hline \multicolumn{4}{|l|}{ NPO } \\
\hline Intervention & $7.00(6.38$ to 7.61$)$ & 5.32 (4.79 to 5.86$)$ & \multirow{2}{*}{$0.14(-0.06$ to 0.33$)$} \\
\hline Control & 6.99 (6.34 to 7.63$)$ & 5.87 (5.31 to 6.42$)$ & \\
\hline \multicolumn{4}{|l|}{ PPO } \\
\hline Intervention & 9.12 (8.57 to 9.67$)$ & 11.33 (10.87 to 11.79$)$ & \multirow{2}{*}{$0.09(-0.11$ to 0.28$)$} \\
\hline Control & 9.18 (8.68 to 9.68$)$ & 11.04 (10.59 to 11.49$)$ & \\
\hline \multicolumn{4}{|l|}{ PSWQ } \\
\hline Intervention & 9.44 (8.88 to 10.00$)$ & 6.49 (5.91 to 7.07$)$ & \multirow{2}{*}{$0.16(-0.04$ to 0.35$)$} \\
\hline Control & $9.76(9.22$ to 10.30$)$ & 7.17 (6.56 to 7.77$)$ & \\
\hline \multicolumn{4}{|l|}{ ISI } \\
\hline Intervention & $12.00(11.19$ to 12.80$)$ & 8.85 (8.14 to 9.57$)$ & \multirow{2}{*}{$0.15(-0.05$ to 0.34$)$} \\
\hline Control & $11.70(10.88$ to 12.51$)$ & 9.64 (8.88 to 10.34$)$ & \\
\hline \multicolumn{4}{|l|}{ PSMS } \\
\hline Intervention & 19.11 (18.64 to 19.59$)$ & 20.80 (20.33 to 21.27$)$ & \multirow{2}{*}{$0.14(-0.05$ to 0.33$)$} \\
\hline Control & $19.22(18.81$ to 19.63$)$ & 20.33 (19.87 to 20.79$)$ & \\
\hline
\end{tabular}

Abbreviations: BADS-SF, Behavioral Activation for Depression Scale-Short Form (total score range, 0-56; higher scores indicate high activation and low avoidance); CES-D, Centre for Epidemiological Studies Depression Scale (total score range, 0-60; higher scores indicate more severe depressive symptoms); HADS-A, Hospital Anxiety and Depression Scale Anxiety Subscale (total score range, 0-21; score 0-7 indicates no anxiety, 8-10 indicates possible anxiety, >11 indicates clinical anxiety disorder); ISI, Insomnia Severity Inventory (total score range, 0-28; higher scores indicate more severe insomnia); PSMS, Pearling and Schooler Mastery Scale (total score range, 0-21; higher scores indicate more mastery); PSWQ, Penn State Worrying Questionnaire, ultra-brief form (total score range, 0-18; higher scores indicate more worry);

SF-12 MCS, 12-Item Short Form Health Survey mental health summary score; SF-12 PCS, 12-Item Short Form Health Survey physical health summary score (SF-12 total scores on the 2 subscales [mental health and physical health] are calculated in such a way that they compare with a norm population with a mean score of 50 and a standard deviation of 10); SPSI-NPO, Social Problem Solving Inventory-Revised, Negative Problem Orientation Subscale (total score range, 0-20; lower scores indicate more negative problem orientation); SPSI-PPO, Social Problem Solving Inventory-Revised, Positive Problem Orientation Subscale (total score range, 0-20; lower scores indicate more positive problem orientation).

Table 3. Health Care Service Use During 12-Month Follow-up Period by Study Condition

\begin{tabular}{|c|c|c|c|c|c|c|}
\hline & \multicolumn{4}{|l|}{ №. (\%) } & \multirow{2}{*}{\multicolumn{2}{|c|}{$\begin{array}{l}\text { Difference in Percentages } \\
\text { Between Study Conditions }(95 \% \mathrm{Cl})^{\mathrm{a}}\end{array}$}} \\
\hline & \multicolumn{2}{|l|}{ Intervention Group } & \multicolumn{2}{|l|}{ Control Group } & & \\
\hline & $\begin{array}{l}\text { 6-Month Follow-up } \\
(\mathrm{n}=152)\end{array}$ & $\begin{array}{l}\text { 12-Month Follow-upc } \\
(\mathrm{n}=130)\end{array}$ & $\begin{array}{l}\text { 6-Month Follow-up } \\
(\mathrm{n}=175)\end{array}$ & $\begin{array}{l}\text { 12-Month Follow-up } \\
(\mathrm{n}=158)\end{array}$ & 6-Month Follow-up & 12-Month Follow-up \\
\hline Primary care clinician & $96(63.2)$ & $79(60.8)$ & $88(50.3)$ & $83(52.5)$ & 12.9 (2 to 23$)$ & $8.3(-3$ to 19$)$ \\
\hline Psychotherapist & $6(3.9)$ & $10(7.7)$ & $17(9.7)$ & $12(7.6)$ & $5.8(0.1$ to 11$)$ & 0.1 ( -6 to 7$)$ \\
\hline Antidepressants & $32(21.1)$ & $27(20.8)$ & 40 (22.9) & $43(27.2)$ & $1.8(-7$ to 11$)$ & $6.4(-4$ to 16$)$ \\
\hline Neurologist & $11(7.2)$ & $10(7.7)$ & $9(5.1)$ & $8(5.1)$ & 2.1 ( -3 to 8$)$ & $2.6(-3$ to 9$)$ \\
\hline Psychiatrist & $3(2)$ & $2(1.5)$ & $8(4.6)$ & $6(3.8)$ & $2.6(-2$ to 7$)$ & 2.3 ( -2 to 7$)$ \\
\hline $\begin{array}{l}\text { Psychosomatic } \\
\text { medicine specialist }\end{array}$ & 0 & $1(0.8)$ & $1(0.6)$ & $2(1.3)$ & 0.6 ( -2 to 3$)$ & 0.5 (-3 to 4$)$ \\
\hline \multicolumn{5}{|c|}{$\begin{array}{l}\text { a Based on Newcombe. }{ }^{41} \\
\text { b Six-month follow-up covering the previous } 3 \text { months as measured with the } \\
\text { TiC-P (Trimbos/iMTA Questionnaire for Costs Associated With } \\
\text { Psychiatric illness). }\end{array}$} & the previous 3 mont & רs as measured with \\
\hline
\end{tabular}


Additionally, the use of antidepressant medication was not an exclusion criterion. Because we excluded those participants with MDD in the previous 6 months, we assumed that we did not include participants treated for depression. However, we cannot rule out that for some participants the web-based intervention was an adjunct to concurrent antidepressant treatment (ie, secondary prevention). Also, we did not measure the uptake of the web-based psychoeducational intervention. Future studies should investigate a possible dose-effect relationship. Eleventh, some unguided web-based interventions for depressive symptoms have been shown to be possibly ineffective. ${ }^{40}$ Because the intervention in this study relied on the use of online trainers, it is therefore possible that unguided web-based interventions would be less effec- tive or ineffective. Studies are needed to evaluate the preventive effects of unguided web-based interventions on the onset of MDD.

\section{Conclusions}

Among patients with subthreshold depression, the use of a web-based guided self-help intervention compared with enhanced usual care reduced the incidence of MDD over 12 months. Further research is needed to understand whether the effects are generalizable to both first onset of depression and depression recurrence as well as efficacy without the use of an online trainer.

\section{ARTICLE INFORMATION}

Correction: This article was corrected online on May 31, 2016, to correct corresponding author information.

Author Affiliations: Division of Online Health Training, Innovation Incubator, Leuphana University Lueneburg, Germany (Buntrock, Ebert, Lehr, Riper, Berking, Cuijpers); Department of Clinical Psychology, EMGO+ Institute for Health and Care Research, VU University, Amsterdam, the Netherlands (Buntrock, Smit, Riper, Cuijpers); Department of Clinical Psychology and Psychotherapy, Friedrich-Alexander-University, Erlangen-Nuremberg, Germany (Ebert, Berking); Department of Public Mental Health, Trimbos Institute (Netherlands Institute of Mental Health and Addiction), Utrecht (Smit); Department of Epidemiology and Biostatistics, EMGO+ Institute for Health and Care Research, VU University Medical Centre, Amsterdam, the Netherlands (Smit); Institute of Telepsychiatry, University of Southern Denmark, Odense (Riper).

Author Contributions: Ms Buntrock had full access to all of the data in the study and takes responsibility for the integrity of the data and the accuracy of the data analysis. Ms Buntrock and Dr Ebert share first authorship. Study concept and design: All authors. Acquisition, analysis, or interpretation of data: Buntrock, Ebert, Smit, Cuijpers. Drafting of the manuscript: Buntrock, Ebert. Critical revision of the manuscript for important intellectual content: All authors.

Statistical analysis: Buntrock, Ebert, Smit. Obtained funding: Berking.

Administrative, technical, or material support: All authors.

Study supervision: Ebert, Lehr, Smit, Riper, Berking, Cuijpers.

Conflict of Interest Disclosures: All authors have completed and submitted the ICMJE Form for Disclosure of Potential Conflicts of Interest. The Leuphana University, Lueneburg, has the exploitation rights of the intervention. The authors will not have a share in any possible license revenues from the Leuphana University Lueneburg. However, Dr Ebert, Dr Lehr, and Dr Berking reported holding shares of the Institute for Online Health Training, which aims to transfer scientific knowledge related to the present research into routine mental health care in Germany. This institute licenses the intervention under study from the Leuphana University, Lueneburg, to provide the intervention within routine preventive services of health insurance companies in Germany. The foundation of such an institute to disseminate findings and products from the research project was the primary aim of the European Union for funding the presented research. Dr Berking reported receiving research grants from the German Ministry of Research and the German Research Association and receiving personal fees from various institutions providing ongoing training for psychotherapists. No other disclosures were reported.

Funding/Support: The associated project of all authors was funded by the European Union (project EFRE: CCI 2007DE161PRO01) and the BARMER GEK (German statutory health insurance company). Role of Funders/Sponsors: The funding bodies had no role in the design and conduct of the study; collection, management, analysis, and interpretation of the data; preparation, review, and approval of the manuscript; and decision to submit the manuscript for publication.

\section{REFERENCES}

1. Vos T, Flaxman AD, Naghavi M, et al. Years lived with disability (YLDs) for 1160 sequelae of 289 diseases and injuries 1990-2010: a systematic analysis for the Global Burden of Disease Study 2010 [published correction appears in Lancet. 2013;381(9867):628]. Lancet. 2012;380(9859): 2163-2196.

2. Kessler RC, Sampson NA, Berglund P, et al. Anxious and non-anxious major depressive disorder in the World Health Organization World Mental Health Surveys. Epidemiol Psychiatr Sci. 2015;24(3): 210-226.

3. Ferrari AJ, Somerville AJ, Baxter AJ, et al. Global variation in the prevalence and incidence of major depressive disorder: a systematic review of the epidemiological literature. Psychol Med. 2013;43(3): 471-481.

4. Mathers CD, Loncar D. Projections of global mortality and burden of disease from 2002 to 2030. PLoS Med. 2006;3(11):e442.

5. Andrews G, Issakidis C, Sanderson K, Corry J, Lapsley H. Utilising survey data to inform public policy: comparison of the cost-effectiveness of treatment of ten mental disorders. Br J Psychiatry. 2004;184:526-533.

6. van Zoonen K, Buntrock C, Ebert DD, et al. Preventing the onset of major depressive disorder: a meta-analytic review of psychological interventions. Int J Epidemiol. 2014;43(2):318-329.

7. Spek V, Cuijpers P, Nyklícek I, Riper H, Keyzer J, Pop V. Internet-based cognitive behaviour therapy for symptoms of depression and anxiety: a meta-analysis. Psychol Med. 2007;37(3):319-328.

8. Ebert DD, Berking M, Cuijpers P, Lehr D, Pörtner $\mathrm{M}$, Baumeister $\mathrm{H}$. Increasing the acceptance of Internet-based mental health interventions in primary care patients with depressive symptoms: a randomized controlled trial. J Affect Disord. 2015; 176:9-17.

9. Radloff LS. The CES-D Scale: a self-report depression scale for research in the general population. Appl Psychol Meas. 1977;1(3):385-401.

10. Lohse MJ, Lorenzen A, Müller-Oerlinghausen B. Psychopharmaka. In: Schwabe U, Pfaffrath D, eds. Arzneiverordnungsreport-Report 2007. Heidelberg, Germany: Springer; 2008:775-813.

11. Kupfer DJ. Long-term treatment of depression. J Clin Psychiatry. 1991;52(suppl):28-34.

12. Bundesärztekammer. Bundesvereinigung $\mathrm{K}$, Fachgesellschaften AdWM. German S3-Guideline/National Disease Management Guideline Unipolar Depression. http://www versorgungsleitlinien.de/themen/depression. 2011. Accessed April 4, 2016.

13. Hakkaart-van Roijen L vSA, Donker M, Tiemens B. Manual Trimbos/iMTA Questionnaire for Costs Associated With Psychiatric Illness (TiC-P). Rotterdam, the Netherlands: Erasmus University; 2002.

14. Lobbestael J, Leurgans $M$, Arntz A. Inter-rater reliability of the Structured Clinical Interview for DSM-IV Axis I Disorders (SCID I) and Axis II Disorders (SCID II). Clin Psychol Psychother. 2011;18 (1):75-79.

15. Crippa JA, de Lima Osório F, Del-Ben CM, Filho AS, da Silva Freitas MC, Loureiro SR. Comparability between telephone and face-to-face structured clinical interview for DSM-IV in assessing social anxiety disorder. Perspect Psychiatr Care. 2008;44 (4):241-247

16. Lyketsos CGNG, Cwi J, Heithoff K, Eaton WW. The Life Chart Interview: a standardized method to describe the course of psychopathology. Int J Methods Psychiatr Res. 1994:4:143-155.

17. Gandek B, Ware JE, Aaronson NK, et al; International Quality of Life Assessment. Cross-validation of item selection and scoring for the SF-12 Health Survey in nine countries: results 
from the IQOLA Project. J Clin Epidemiol. 1998;51 (11): $1171-1178$

18. Zigmond AS, Snaith RP. The Hospital Anxiety and Depression Scale. Acta Psychiatr Scand. 1983; 67(6):361-370.

19. D'Zurilla TJ, Nezu AM, Maydeu-Olivares $A$. Social Problem-Solving Inventory-Revised (SPSI-R). New York, NY: Multi-Health Systems; 2002.

20. Manos RC, Kanter JW, Luo W. The Behavioral Activation for Depression Scale-Short Form: development and validation. Behav Ther. 2011:42 (4):726-739.

21. Pearlin LI, Schooler C. The structure of coping. J Health Soc Behav. 1978;19(1):2-21.

22. Berle D, Starcevic V, Moses K, Hannan A, Milicevic D, Sammut P. Preliminary validation of an ultra-brief version of the Penn State Worry Questionnaire. Clin Psychol Psychother. 2011;18(4): 339-346.

23. Bastien $\mathrm{CH}$, Vallières $\mathrm{A}$, Morin $\mathrm{CM}$. Validation of the Insomnia Severity Index as an outcome measure for insomnia research. Sleep Med. 2001;2 (4):297-307.

24. Allart-van Dam E, Hosman CM, Hoogduin CA, Schaap CP. Prevention of depression in subclinically depressed adults: follow-up effects on the "Coping with Depression" course. J Affect Disord. 2007;97 (1-3):219-228.

25. Hagan R, Evans SF, Pope S. Preventing postnatal depression in mothers of very preterm infants: a randomised controlled trial. BJOG. 2004 111(7):641-647.

26. Schulz KF, Altman DG, Moher D. CONSORT 2010 statement: updated guidelines for reporting parallel group randomised trials. J Pharmacol Pharmacother. 2010;1(2):100-107.

27. Kleinbaum DG, Klein M. Survival Analysis: A Self-learning Text. 3rd ed. New York, NY: Springer: 2012.

28. Grambsch P, Therneau T. Proportional hazards tests and diagnostics based on weighted residuals. Biometrika. 1994;81(3):515-526.

29. Altman DG, Andersen PK. Calculating the number needed to treat for trials where the outcome is time to an event. BMJ. 1999;319(7223) 1492-1495.

30. Buntrock C, Ebert D, Lehr D, et al. Effectivenes of a web-based cognitive behavioural intervention for subthreshold depression: pragmatic randomised controlled trial. Psychother Psychosom 2015;84(6):348-358.

31. Stata Statistical Software: Release 13 [computer program]. College Station, TX: StataCorp; 2013.

32. Otero P, Smit F, Cuijpers P, Torres A, Blanco V Vázquez FL. Long-term efficacy of indicated prevention of depression in non-professional caregivers: randomized controlled trial. Psychol Med. 2015;45(7):1401-1412.

33. Willemse GR, Smit F, Cuijpers P, Tiemens BG Minimal-contact psychotherapy for sub-threshold depression in primary care: randomised trial. $\mathrm{Br} J$ Psychiatry. 2004;185:416-421.

34. Zhang $X$, Lewis $G$, Araya $R$, et al. Prevention of anxiety and depression in Chinese: a randomized clinical trial testing the effectiveness of a stepped care program in primary care. J Affect Disord. 2014 169:212-220.

35. de Jonge P, Hadj FB, Boffa D, et al. Prevention of major depression in complex medically ill patients: preliminary results from a randomized controlled trial. Psychosomatics. 2009;50(3):227233.

36. Cookson J, Gilaberte I, Desaiah D, Kajdasz DK. Treatment benefits of duloxetine in major depressive disorder as assessed by number needed to treat. Int Clin Psychopharmacol. 2006;21(5): 267-273.

37. Mack $S$, Jacobi F, Gerschler $A$, et al. Self-reported utilization of mental health services in the adult German population-evidence for unmet needs? results of the DEGS1-Mental Health Module (DEGS1-MH). Int J Methods Psychiatr Res. 2014;23 (3):289-303.

38. Cuijpers $P$, van Straten $A$, Warmerdam $L$, van Rooy MJ. Recruiting participants for interventions to prevent the onset of depressive disorders: possible ways to increase participation rates. BMC Health Serv Res. 2010;10:181.

39. Zwarenstein M, Treweek S, Gagnier JJ, et al CONSORT Group; Pragmatic Trials in Healthcare (Practihc) Group. Improving the reporting of pragmatic trials: an extension of the CONSORT statement. BMJ. 2008:337:a2390.

40. Gilbody S, Littlewood E, Hewitt C, et al; REEACT Team. Computerised cognitive behaviour therapy (cCBT) as treatment for depression in primary care (REEACT trial): large scale pragmatic randomised controlled trial. BMJ. 2015;351:h5627.

41. Newcombe RG. Interval estimation for the difference between independent proportions comparison of eleven methods [published correction appears in Stat Med. 1999;18(10):1293] Stat Med. 1998;17(8):873-890. 